\title{
Vibrational Power Harvester Based on Lithium Niobate Bidomain Plate
}

\author{
I.V. Kubasov*, A.M. Kislyuk, M.D. Malinkovich, A.A. Temirov, S.V. Ksenich, \\ D.A. Kiselev, A.S. Bykov and Y.N. Parkhomenko \\ National University of Science and Technology MISIS, Leninskiy prosp. 4, 119049 Moscow, Russia
}

\begin{abstract}
In this paper we report the design of piezoelectric vibrational energy harvester based on single crystalline bidomain lithium niobate plate. Energy conversion was obtained by using cantilever fastened piezoelectric plate with seismic mass at the free end. Output voltage was measured depending on seismic mass and storage capacitance. Maximum voltage of $35 \mathrm{mV}$ was measured with vibrational excitation by an industrial air compressor.
\end{abstract}

DOI: 10.12693/APhysPolA.134.90

PACS/topics: power harvester, lithium niobate, bidomain crystal, vibrational-to-electric energy conversion

\section{Introduction}

Usage of piezoelectric effect for generation of electrical energy is a steadily growing trend of modern small-sized energetics [1-4]. Recent studies in this field were devoted to harvesting energy from vibrations of different industrial equipment [1], pressure of humans' steps [5] and vehicle wheels [6], water flow [7] and some other sources. It was stated that density of energy harvested by piezoelectric generators might be at least twice higher than the that of electrostatic and electromagnetic power harvesters [8]. Generally, the reason of the attention to piezoelectric energy-harvesting devices is in their simplicity, relatively low cost in mass production and long lifecycle.

The most common materials for piezoelectric power generators are piezoceramics with different compositions, polyvinylidene fluoride (PVDF) and its copolymers, and relaxor single crystals [9-12]. However, the aforementioned materials are unstable at high temperatures due to their relatively low Curie point. At the same time, there is a necessity of construction of effective lowpower generators, which can utilize vibration energy at high temperatures in order to supply autonomous equipment, e.g. sensors.

There are many piezoelectric materials, which can withstand high temperatures, but most often, their piezoelectric coefficients are too low and due to this reason cannot transform mechanical energy into electricity effectively.

It is possible to increase the efficiency of energy harvesters based on piezoelectric single crystal by using bimorph or bicrystal structure, however the presence of the adhesive layer nullifies thermal stability of such construction and decreases the efficiency at room temperature.

*corresponding author; e-mail: kubasov.ilya@gmail.com
On the other hand, it is known that there is a possibility to create bimorph structure in plates of single crystalline ferroelectrics of lithium niobate $\left(\mathrm{LiNbO}_{3}\right)[13,14]$ and lithium tantalate $\left(\mathrm{LiTaO}_{3}\right)$ [15] without glue or any intergranular boundaries. Today, this problem can be solved by three different ways: by annealing the crystal near Curie point in order to cause out-diffusion of $\mathrm{Li}_{2} \mathrm{O}$ [13-16]; by electrothermal annealing [17, 18]; by annealing in non-uniform thermal field [15, 19]. The result of these processes is a single crystalline plate with two oppositely oriented ferroelectric domains (the bidomain structure).

Previously such bidomain structures were investigated as perspective replacement for $\mathrm{PZT}\left(\mathrm{PbZr}_{x} \mathrm{Ti}_{1-x} \mathrm{O}_{3}\right)$ based piezoelectric actuators due to outstanding properties like absence of electromechanical hysteresis, creep and high thermal stability. There also was an attempt to create a piezoelectric generator based on $\mathrm{LiNbO}_{3}$ bidomain single crystal [20], but authors have conducted their experiments in laboratory conditions and used single impacts in order to generate electrical energy up to $10 \mathrm{~V}$.

In this study, we have attempted to estimate the possibility to use cantilevered bidomain $\mathrm{LiNbO}_{3}$ plate for mechanical energy harvesting from industrial source of vibrations (air compressor). Several different seismic masses were applied to the end of the bender in order to change the resonance frequency of the device. We also used different capacitors for decreasing voltage fluctuations and to store energy.

\section{Experimental}

In the experiment, single crystalline single-domain plates of lithium niobate were used (commercially available from Elan Ltd., St.-Petersburg, Russia). In order to maximize working piezoelectric coefficient $y+128^{\circ}$ oriented wafers were selected. From the wafer, we have cut a rectangular sample with the area of $5 \times 37 \mathrm{~mm}^{2}$ and the thickness of $0.5 \mathrm{~mm}$, with the long side perpendicular to the $x$ axis. The active (non-clamped) length was selected to be $32 \mathrm{~mm}$. 
Bidomain ferroelectric structure was formed into the plate by using the stationary external heating method [19], where a non-uniform thermal field was provided by rapid infrared heating-cooling system ULVAC VHC-P610. Tantalum electrodes with thickness of $100 \mathrm{~nm}$ were deposited on the crystal faces by DC magnetron sputtering. The fastened end of the bidomain plate was not covered with electrodes.

In order to create cantilevered bending vibrator we used a base made of rectangular polycrystalline alumina wafer with the system of fastening plates at one of its ends, as is schematically shown in Fig. 1a. In order to fix the bidomain crystal by these plates we used two stainless screws. The output ac voltage was transferred to the external circuit by two conductive strips made of aluminum foil (Fig. 1b).

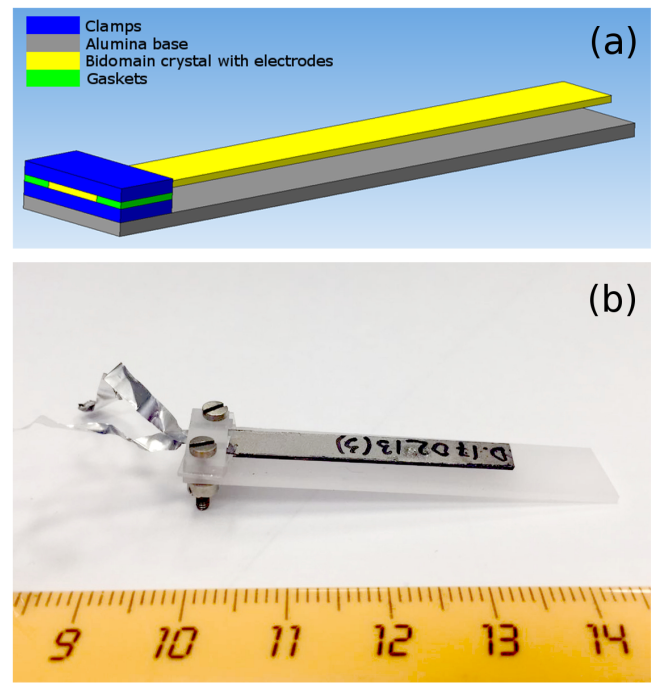

Fig. 1. (a) Schematic representation of the vibrational energy harvester; (b) photograph of the harvester.

For the experiment, we have assembled a simple circuit with a diode bridge rectifier and a capacitor (Fig. 2a). The input of the circuit was connected to electrodes of the bidomain plate. The output voltage was measured by an oscilloscope (Agilent DSO-X 3032A) with 1:10 passive probe (full resistance of the load was equal to $10 \mathrm{M} \Omega$ ). In order to excite vibrations we used an UDT-2525 air compressor. The vibrational energy harvester with the circuit was bonded on the top of the compressor where the strongest vibrations were observed. The appearance of our testing setup is shown in Fig. $2 \mathrm{~b}$.

Measurements were started with the empty compressor tank, thus the beginning state was the same for the each measurement. Every measurement was stopped when the signal at the oscilloscope screen remained unaltered for ten seconds. For changing the resonant frequency, several seismic masses $(0.15 \mathrm{~g}, 1.18 \mathrm{~g}$ and $3.72 \mathrm{~g})$ were applied to the free end of the cantilever. We also were changing the external storage capacitor by soldering different variants of it to the circuit board.
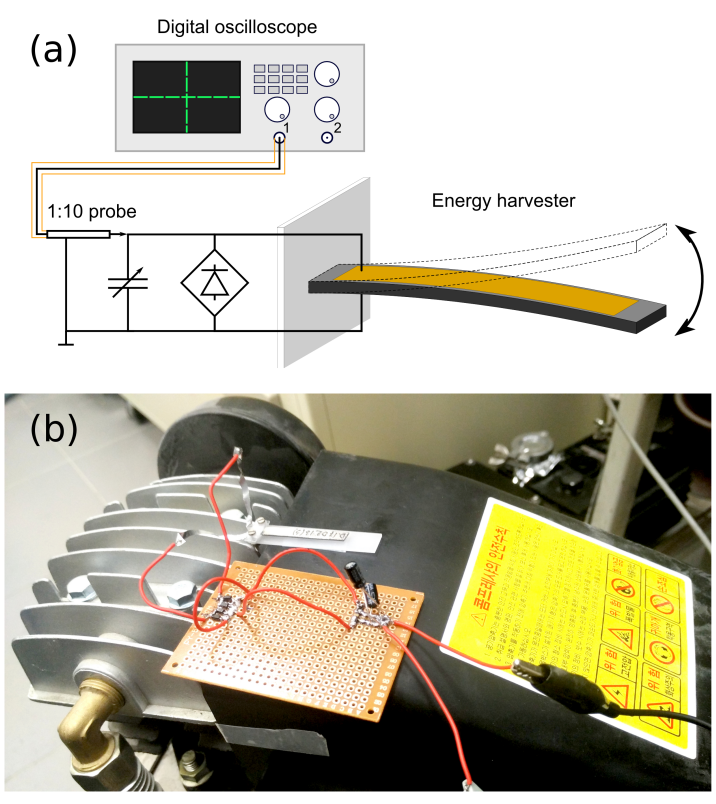

Fig. 2. (a) The diagram of the experimental tools; (b) photograph of the testing setup of the vibrational energy harvesting device.

\section{Results and discussion}

Figure 3a shows measured dependence of the output voltage on the load applied to the free end of the cantilever. The data indicates that the best energy conversion is achieved when the seismic mass of $1.18 \mathrm{~g}$ is attached to the free end of the cantilever. It is clear, that this optimum mass is related only to the vibrational spectrum of this air compressor. Therefore, we can conclude, that the seismic mass should be accurately selected for each specific vibrational source. Moreover, the vibrational spectrum of a real source of mechanical oscillations may changed during the operation, e.g. due to changing of energy consumption by a motor. This feature of real devices is also important and must be considered during the development of a power harvester.

Figure 3b plots dependence of output voltage on the storage capacitance, placed after the diode bridge in the circuit during the experiment. Dispersion of points at the left side of figure is caused by the pulsations that are not filtered by a small capacitance. Here we also can see that the best results are achieved when an optimal capacitor is used $(\approx 30 \mu \mathrm{F})$. It is clear that a too small capacitance charges and discharges too fast, and thus the average voltage is relatively small. A big capacitor $(1000 \mu \mathrm{F})$ cannot be fully charged by the vibrational energy harvester thus, there are no pulses, but the output voltage is also relatively small.

It should be also mentioned that we were not able to obtain output voltages higher than dozens of millivolts in contrast to Funasaka's paper [20], where values of order of volts were observed in the harvester with close geometrical characteristics. This could be explained by utilizing 

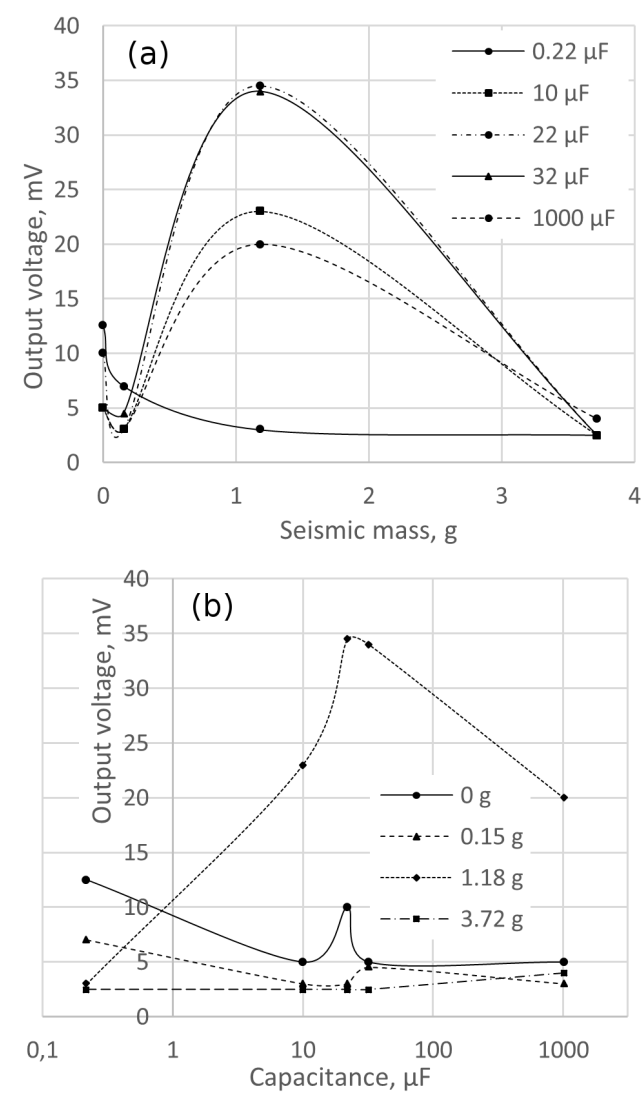

Fig. 3. (a) Dependence of the output voltage on the load applied to the free end of the cantilever for different storage capacitances; (b) dependence of the output voltage on the storage capacitance for different seismic masses.

another type of vibrational source in our study. Due to presence of many different frequencies and directions of movements in vibrational spectrum of the air compressor, this source exhibits a more "realistic" behavior, than a single impacts at the free end of the cantilever. Moreover, the high-resistance load of $10 \mathrm{M} \Omega$ is small enough to discharge drastically the storage capacitor from the peak voltage. Thus, serious improvements of design are necessary in order to create a highly effective power generator.

\section{Conclusions}

To conclude, vibrational energy harvesting device based on single crystalline bidomain plate of lithium niobate was assembled and tested. Experiments were carried out using an air compressor as the industrial source of vibration. Maximum voltage of $35 \mathrm{mV}$ was obtained with $1.18 \mathrm{~g}$ seismic mass and $30 \mu \mathrm{F}$ storage capacitor. Optimum mass and capacitor should be chosen for each type of vibrational sources due to differences of the spectra of mechanical oscillations. The output voltage of the studied vibrational energy harvester is three orders of magnitude lower than that of the impact-excited harvester from [20]. The design of harvester should be improved in order to utilize it in commercial applications.

\section{Acknowledgments}

The study was supported by the Ministry of Education and Science of the Russian Federation (Federal Targeted Programme for Research and Development in Priority Areas of Development of the Russian Scientific and Technological Complex for 2014-2020) (Project ID RFMEFI58716X0035)

\section{References}

[1] H.A. Sodano, D.J. Inman, G. Park, Shock and Vibration Digest 36(3), 197 (2004).

[2] S.R. Anton, H.A. Sodano, Smart Materials and Structures 16(3), R1 (2007).

[3] H.S. Kim, J.H. Kim, J.A. Kim, International Journal of Precision Engineering and Manufacturing 12(6), 1129 (2011).

[4] U. Khan, R. Hinchet, H. Ryu, S.W. Kim, APL Materials 5, 073803 (2017).

[5] N.S. Shenck, J.A. Paradiso, IEEE Micro 21(3), 30 (2001).

[6] G. Manla, N.M. White, J. Tudor, TRANSDUCERS 2009 - International Solid-State Sensors, Actuators and Microsystems Conference, IEEE 2009, p. 1389.

[7] S. Pobering, N. Schwesinger, MEMS, NANO and Smart Systems, 2004. ICMENS 2004. Proceedings, IEEE 2004, p. 480.

[8] S. Priya, Journal of Electroceramics 19(1), 167 (2007).

[9] E.S. Leland, P.K. Wright, Smart Materials and Structures 15, 1413 (2006).

[10] M.A. Karami, D.J. Inman, Appl. Phys. Lett. 100, 042901 (2012).

[11] C.S. Lee, J. Joo, S. Han, J.H. Lee, S.K. Koh, Synthetic Metals 152, 49 (2005).

[12] B. Ren, Y.Zhang, Q. Zhang, X. Li, W. Di, X. Zhao, H. Luo, S.W. Or, Applied Physics A 100, 125 (2010).

[13] K. Nakamura, H. Ando, H. Shimizu, Japan. J. Appl. Phys. 26(S2), 198 (1987).

[14] K. Nakamura, H. Shimizu, Ferroelectrics 93, 211 (1989).

[15] I.V. Kubasov, A.M. Kislyuk, A.S. Bykov, M.D. Malinkovich, R.N. Zhukov, D.A. Kiselev, S.V. Ksenich, A.A. Temirov, N.G. Timushkin, Y.N. Parkhomenko, Crystallography Reports 61, 258 (2016).

[16] V.D. Kugel, G. Rosenman, Appl. Phys. Lett. 62, 2902 (1993)

[17] V.V. Antipov, A.S. Bykov, M.D. Malinkovich, Y.N. Parkhomenko, Ferroelectrics 374, 65 (2008).

[18] M.D. Malinkovich, A.S. Bykov, I.V. Kubasov, D.A. Kiselev, S.V. Ksenich, R.N. Zhukov, A.A. Temirov, N.G. Timushkin, Y.N. Parkhomenko, Russian Microelectronics 45, 582 (2016).

[19] A.S. Bykov, S.G. Grigoryan, R.N. Zhukov, D.A. Kiselev, S.V. Ksenich, I.V. Kubasov, M.D. Malinkovich, Y.N. Parkhomenko, Russian Microelectronics 43, 536 (2014).

[20] T. Funasaka, M. Furuhata, Y. Hashimoto, K. Nakamura, Ultrasonics Symposium 1998. Proceedings, IEEE 1998, p. 959. 